\title{
Outcomes of Patients With Cocaine Induced Chest Pain in An Inner City Hospital
}

\author{
Moustapha Atoui ${ }^{\mathrm{a}}$, Nadia Fida ${ }^{\mathrm{a}}$, Suresh Kumar Nayudua, \\ Mariela Glandt ${ }^{\mathrm{a}}$, Sridhar Chilimuri ${ }^{\mathrm{a}, \mathrm{b}}$
}

\begin{abstract}
Background: Cocaine induced chest pain is a major reason for admission in Safety Net Hospitals in the United States. The majority of patients admitted undergo extensive work-up leading to enormous economic burden. We hypothesize that in individuals with low risk, cocaine does not further increase adverse cardiovascular outcomes.
\end{abstract}

Methods: We conducted a retrospective chart review of all patients admitted with chest pain to our hospital between 07/01/09 and 06/30/10. We excluded patients with modifiable risk factors for coronary artery disease (CAD). The study population was divided into cocaine and non-cocaine group based on urine drug screen. We analyzed data including demographic, laboratory, cardiac testing, detection of CAD, length of stay and mortality rates.

Results: A total of 426 individuals matched our inclusion and exclusion criteria and were considered to have no known modifiable cardiac risk factors; 54 in cocaine group and 372 in non-cocaine group. Based on physician discretion, $41(76 \%)$ in the cocaine group and 239(64\%) in the non-cocaine group underwent various modalities of cardiac testing. Cardiac testing was positive in $6(2.5 \%)$ patients in non-cocaine group and none in the cocaine group ( $\mathrm{p}=0.597)$. There was no significant difference between length of stay and inhospital mortality between the two groups.

Conclusions: In individuals at low risk for CAD, cocaine use resulted in higher rate of cardiac testing. However, there is no difference in prevalence of CAD and in-hospital mortality between the

Manuscript accepted for publication October 14, 2011

${ }^{\mathrm{a}}$ Department of Medicine, Bronx Lebanon Hospital Center, Affiliated with Albert Einstein College of Medicine, Bronx, New York, USA

${ }^{\mathrm{b}}$ Corresponding author: Sridhar Chilimuri, Department of Medicine, 1650 Selwyn Avenue, 10th Floor, Bronx, New York-10457, USA. Email: chilimuri@bronxleb.org

doi:10.4021/cr103w two groups. We conclude that cocaine does not increase adverse outcomes in patients with low risk for CAD.

Keywords: Cocaine; Chest Pain; Outcome

\section{Introduction}

Cocaine is a significant problem in urban hospitals, accounting for the most encountered emergency department (ED) visits related to substance abuse in the United States [1, 2]. From 1999 to 2002, the number of these visits increased by $47 \%$ [3]. Due to its inherent pharmacokinetic properties, cocaine is known to cause several cardiovascular complications, including myocardial infarction, myocarditis, myopathy, arrhythmias, coronary artery aneurysm formation, stroke, and aortic dissection [4]. These complications appear to be independent of dose and route of administration [5]. Most of the risk of myocardial infarction appears to be within the first few hours following cocaine use [6-8].

In current practice, these patients using cocaine are managed as if they had modifiable risk factors for CAD. These patients also have decreased likelihood of compliance with outpatient follow-up [9]. For these two reasons, most of this population undergoes in-patient cardiac testing once admitted.

The management of these patients, however, is based on limited data, making it a challenge for physicians to riskstratify them. We were interested in studying whether cocaine should be regarded as a traditional risk factor for heart disease in the subgroup of patients who present to the ER with chest pain with no other risk factors other than cocaine use.

\section{Methods}

We conducted a retrospective chart review of consecutive patients admitted with chest pain to Bronx Lebanon Hospital Center (BLHC) between July 1, 2009 and June 30, 2010. Electronic medical records were reviewed for age, gender, race, 
Table 1. Characteristics of Total Study Group

\begin{tabular}{llll}
\hline & Cocaine group & Non-cocaine group & P-value \\
\hline $\begin{array}{l}\text { Number of subjects } \\
\text { Gender }\end{array}$ & $54(13 \%)$ & $372(87 \%)$ & 0.19 \\
$\quad$ Female & $22(41 \%)$ & $191(51 \%)$ & 0.003 \\
$\quad 32(59 \%)$ & $181(49 \%)$ & \\
Male & & & $5(1 \%)$ \\
$\quad$ Asian & $0(0 \%)$ & $110(30 \%)$ & $4(1 \%)$ \\
$\quad$ African American & $29(54 \%)$ & $242(65 \%)$ & 0.47 \\
$\quad$ Caucasian & $2(3.5 \%)$ & $11(3 \%)$ & 0.89 \\
$\quad$ Hispanic & $22(41 \%)$ & $43(12)$ & 0.07 \\
$\quad$ Others & $1(1.5 \%)$ & $28(6)$ & 1 \\
Age, Mean (SD) years & $44(10)$ & $2.4(2.1-2.7)$ & 0.12 \\
BMI (SD) kg/m ${ }^{2}$ & $28(6)$ & 3 & $239(64 \%)$ \\
Length of stay, mean (95\% CI) days & $3(2.4-3.8)$ & 0 & \\
Mortality & $41(76 \%)$ & & \\
Cardiac testing & &
\end{tabular}

body mass index (BMI kg/m²), past medical history, length of stay in days (LOS) and in-hospital mortality. Laboratory data including serum cardiac markers Creatinine phosphokinase (CPK), Creatinine kinsase-MB (CK-MB), Troponin$\mathrm{T}$ and urine toxicology screen was extracted. Records were also reviewed for cardiac testing including echocardiogram, stress tests (nuclear stress test, stress echocardiography and exercise stress test) and cardiac catheterization.

We excluded patients who were admitted with ST elevation myocardial infarction (STEMI), Non-ST elevation myocardial infarction (NSTEMI), and patients who had traditional risk factors for heart disease, including patients with medical history of coronary artery disease (CAD), cardiomyopathy, hypertension, diabetes mellitus, dyslipidemia and active smoking. We also reviewed cardiac work-up done prior to our study dates to exclude any patient with known $\mathrm{CAD}$ and/or chronic systolic congestive heart failure. This was done to exclude patients with atherosclerotic disease because it would make the group not only high risk for further ischemic events, but also because of the reported higher incidence of cocaine-associated cardiovascular events with coexisting coronary artery disease [6].

CAD was defined as luminal obstruction of $50 \%$ or more of coronary arteries and their major branches on cardiac catheterization [10]. Cardiomyopathy was defined as LV ejection fraction less than $40 \%$ irrespective of etiology. Abnormal echocardiogram was defined as systolic dysfunction with ejection fraction $<40 \%$, Grade II and above diastolic dysfunction, or any wall motion abnormality. Nuclear stress test was defined as abnormal when reported as ischemic defect, scar, or transient ischemic dilatation. Positive stress echocardiogramPositive stress echocardiogram is defined as abnormal electrocardiogram and wall motion abnormality reported on test result. Exercise stress test was done using Bruce protocol.

This low risk cohort was then divided into two groups based on positive or negative urine toxicology screen for cocaine.

The data was collected as a Performance Improvement Project in the Department of Medicine at BLHC.

\section{Statistical analysis}

The data was analyzed using SAS JMP 8 version [11]. Mean and standard deviation are calculated for demographics and clinical characteristics of the patients using standard methods. The length of stay (LOS) was compared between two groups by two tailed t-Test for unequal variances. In-hospital mortality between the two groups was compared by Chisquare and Fisher's exact t-Test and plotted as contingency analysis on a mosaic plot. Statistical significance was defined as $\mathrm{P}<0.05$.

\section{Results}

In the selected review period of one year, a total of 2783 patients were admitted for chest pain. Of these patients, 
Table 2. Characteristics of Individuals Who Underwent Cardiac Testing

\begin{tabular}{llll}
\hline & Cocaine group & Non-cocaine group & P-value \\
\hline $\begin{array}{l}\text { Number of subjects } \\
\text { Gender }\end{array}$ & $41(76 \%)$ & $239(64 \%)$ & 0.12 \\
$\quad$ Female & $18(44 \%)$ & $126(53 \%)$ & 0.31 \\
$\quad$ Male & $23(56 \%)$ & $113(47 \%)$ & 0.01 \\
Race & & & \\
$\quad$ Asian & $0(0 \%)$ & $4(1.5 \%)$ & $71(30 \%)$ \\
$\quad$ African American & $23(56 \%)$ & $2(0.5 \%)$ & $155(65 \%)$ \\
$\quad 1(2 \%)$ & $7(3 \%)$ & 0.82 \\
$\quad$ Caucasian & $17(42 \%)$ & $44(12)$ & 0.6 \\
$\quad$ Others & $0(0 \%)$ & $28(6.5)$ & 0.05 \\
Age, Mean (SD) years & $45(9)$ & $2.4(2.3-3.0)$ & 1 \\
BMI (SD) kg/meter square & $29(7)$ & $3(1 \%)$ & 0.6 \\
Length of stay, mean (95\% CI) days & $3.5(2.7-4.3)$ & $6(2.5 \%)$ & \\
Mortality & 0 & $0(0 \%)$ & \\
Positive Cardiac testing & &
\end{tabular}

289 (10.4\%) tested positive for cocaine on urine toxicology screen. Of the total admissions, 2357 (85\%) were excluded based on the exclusion criteria mentioned above. The remaining cohort of 426 (15\%) patients was divided into two groups based on the result of urine drug screen; 54 (13\%) were positive for cocaine and 372 (87\%) tested negative for cocaine on urine drug screen. Mean age (SD) in cocaine group was $44( \pm 10)$ years and in non-cocaine group $43( \pm 12)$ years $(\mathrm{P}=0.47)$. Both groups also had equal distribution in mean BMI of $23( \pm 6) \mathrm{kg} / \mathrm{m}^{2}(\mathrm{P}=0.89)$ (Table 1). However, cocaine positive group had more males (59\%) when compared to (49\%) in non-cocaine group ( $\mathrm{P}=0.19)$, more African Americans (54\%) when compared to (30\%) in non-cocaine group, less Hispanics (41\%) when compared to $(65 \%)$ in non-cocaine group $(\mathrm{P}=0.003)$. Also noted was, individuals in cocaine group were more likely to undergo cardiac testing (76\%) than the non-cocaine group (64\%) (P $=0.12$ ).

The group that underwent cardiac testing also showed preponderance towards male gender (56\%) in cocaine group when compared to $(47 \%)$ in non-cocaine group $(\mathrm{P}=0.31)$ and African American race (56\%) in cocaine group when compared to $(30 \%)$ in non-cocaine group $(\mathrm{P}=0.01)$. None of the patients in cocaine group had a positive cardiac test result, whereas 6 patients had abnormal cardiac testing in general group $(\mathrm{P}=0.6)$ (Table 2$)$.

The mean length of stay (LOS) (95\% confidence interval, CI) was higher in cocaine group, 3 days (2.4 - 3.8) when compared to non-cocaine group 2.4 days (2.1 - 2.7) (P
$=0.07$ ), though not statistically significant. LOS increased even further in cocaine positive group who underwent cardiac testing, 3.5 days (2.7 - 4.3) when compared to, 2.6 days ( 2.3 - 3.0) in the non-cocaine group $(\mathrm{P}=0.05)$.

There were no deaths in cocaine positive group whereas 3 patients died during their hospital stay in the control group $(\mathrm{P}=1)$. Out of these, one patient died of metastatic colon carcinoma and sepsis and two patients died of metastatic lung carcinoma. There were no deaths from cardiovascular causes during hospital stay.

\section{Discussion}

In this study we examined patients with no modifiable cardiovascular risk factors who presented to our ER with complaints of chest pain who were found to test positive for cocaine on a urine toxicology screen. Cocaine is traditionally considered as a risk factor for heart disease and frequently leads to further cardiac testing. Our study, however, showed that in low risk patients further testing may unnecessary since no difference was found between the cocaine and noncocaine groups.

During our study period, ten percent of patients presenting to the ER with complaints of chest pain were found to actively be using cocaine. This statistic is consistent with the data showing that $85 \%$ of cocaine users are centered in urban areas [2], particularly inner city areas like the Bronx.

Our study differs from other studies in that we report 
a higher mean age (44 years) in patients using cocaine presenting with chest pain [12-16]. The younger age of presentation in other studies (mean age less than 40 years) confers them a null or protective factor by Framingham CAD risk at 10 years. The higher baseline risk of our population still did not warrant further cardiac testing.

Studies show low rates of MI (Myocardial Infarction) and mortality overall in cocaine users. Weber et al [14] reported incidence of AMI to be about $6 \%$ with cocaine use in the cohort that has traditional risk factors for CAD, but no complication occurred beyond 12 hrs of presentation with $100 \%$ survival at discharge. A more recent work by the same authors [12] showed that none of the patients developed cardiovascular complications during the observation period.

Zimmerman et al found that 3 of 48 cocaine-associated chest pain patients $(6 \%)$ who were admitted to the Coronary Care Units (CCU) sustained an Acute Myocardial Infarction [17]. Gitter et al reported no acute coronary events in their cohort of 101 cocaine-associated chest pain patients admitted to monitored beds [18]. However these studies did not differentiate between low and high risk patients.

A scientific statement from American Heart Association [1] on Management of Cocaine-Associated Chest Pain and Myocardial Infarction recommends optional stress testing for low to moderate risk individuals after an uneventful 9-12 hours observation period. Our study suggests that this additional testing may be unnecessary, since our low risk cocaine cohort had no excess adverse events.

Our study is limited by the lack of long term follow-up. Furthermore, the modalities used for cardiac testing varied based on clinician discretion. Larger prospective studies will need to be done to further ascertain cocaine's role as a risk factor for CAD in both low and high risk populations.

\section{Grant Support}

None.

\section{Financial Disclosure}

None.

\section{References}

1. McCord J, Jneid H, Hollander JE, de Lemos JA, Cercek B, Hsue P, Gibler WB, et al. Management of cocaineassociated chest pain and myocardial infarction: a scientific statement from the American Heart Association Acute Cardiac Care Committee of the Council on Clinical Cardiology. Circulation. 2008;117(14):1897-1907.

2. Johnston, L. D., O’Malley, P. M., Bachman, J. G.,
\& Schulenberg, J. E. (2006). Monitoring the Future national survey results on drug use, 1975-2005: Volume I, Secondary school students (NIH Publication No. 065883). Bethesda, MD: National Institute on Drug Abuse.

3. Substance Abuse and Mental Health Services Administration, Office of Applied Studies. Emergency Department Trends From the Drug Abuse Warning Network, Final Estimates 1995-2002, DAWN Series: D-24, DHHS Publication No. (SMA) 03-3780, Rockville, MD, 2003.

4. Cregler LL. Adverse health consequences of cocaine abuse. J Natl Med Assoc. 1989;81(1):27-38.

5. Kloner RA, Rezkalla SH. Cocaine and the heart. N Engl J Med. 2003;348(6):487-488.

6. Jones JH, Weir WB. Cocaine-associated chest pain. Med Clin North Am. 2005;89(6):1323-1342.

7. Hollander JE, Henry TD. Evaluation and management of the patient who has cocaine-associated chest pain. Cardiol Clin. 2006;24(1):103-114.

8. Goel P, Flaker GC. Cardiovascular complications of cocaine use. N Engl J Med. 2001;345(21):1575-1576.

9. Gottheil E, Sterling RC, Weinstein SP. Pretreatment dropouts: characteristics and outcomes. J Addict Dis. 1997;16(2):1-14.

10. Patel MR, Dehmer GJ, Hirshfeld JW, Smith PK, Spertus JA. ACCF/SCAI/STS/AATS/AHA/ASNC 2009 Appropriateness Criteria for Coronary Revascularization: a report by the American College of Cardiology Foundation Appropriateness Criteria Task Force, Society for Cardiovascular Angiography and Interventions, Society of Thoracic Surgeons, American Association for Thoracic Surgery, American Heart Association, and the American Society of Nuclear Cardiology Endorsed by the American Society of Echocardiography, the Heart Failure Society of America, and the Society of Cardiovascular Computed Tomography. J Am Coll Cardiol. 2009;53(6):530-553.

11. JMP, Version 8. SAS Institute Inc., Cary, NC, 19892011.

12. Weber JE, Shofer FS, Larkin GL, Kalaria AS, Hollander JE. Validation of a brief observation period for patients with cocaine-associated chest pain. N Engl J Med. 2003;348(6):510-517.

13. Hoey J. Cocaine-associated chest pain in the emergency department. CMAJ. 2003;168(8):1017.

14. Weber JE, Chudnofsky CR, Boczar M, Boyer EW, Wilkerson MD, Hollander JE. Cocaine-associated chest pain: how common is myocardial infarction? Acad Emerg Med. 2000;7(8):873-877.

15. Kushman SO, Storrow AB, Liu T, Gibler WB. Cocaineassociated chest pain in a chest pain center. Am J Cardiol. 2000;85(3):394-396, A310.

16. Hollander JE, Hoffman RS, Gennis P, Fairweather P, DiSano MJ, Schumb DA, Feldman JA, et al. Prospective multicenter evaluation of cocaine-associated chest 
pain. Cocaine Associated Chest Pain (COCHPA) Study Group. Acad Emerg Med. 1994;1(4):330-339.

17. Zimmerman JL, Dellinger RP, Majid PA. Cocaine-associated chest pain. Ann Emerg Med. 1991;20(6):611-615.
18. Gitter MJ, Goldsmith SR, Dunbar DN, Sharkey SW. Cocaine and chest pain: clinical features and outcome of patients hospitalized to rule out myocardial infarction. Ann Intern Med. 1991;115(4):277-282. 